DOI: 10.1002/ ((please add manuscript number))

Article type: Full Paper

\title{
Specific biosensing using DNA aptamers and nanopores
}

Jinglin Kong ${ }^{+}$, Jinbo Zhu', Kaikai Chen and Ulrich F. Keyser*

$\left.{ }^{+}\right]$These authors contributed equally to this work.

Dr. Jinglin Kong, Dr. Jinbo Zhu, Dr. Kaikai Chen, Prof. Ulrich F. Keyser

Cavendish Laboratory, University of Cambridge, JJ Thomson Ave, Cambridge, CB30HE, United Kingdom

E-mail: ufk20@cam.ac.uk

Keywords: Nanopore, Aptamer, Multiplexed sensing, DNA carrier, Physiological condition

Abstract: The multiplexed biosensing of target molecules with high specificity and accuracy is of fundamental importance in both biological research and medical diagnostics. In this paper we extend the working range of our recent nanopore-DNA carrier based method by introducing a two-step assay using specific DNA aptamers. A signal translation step allows for binding of the target in physiological conditions before the nanopore measurements. Using protein encoded DNA carriers, we demonstrate the simultaneous detection of 3 targets spanning several orders of magnitude in molecular weight. Our single-molecule method may be integrated into nanopore sensing devices for future applied research and point of care applications.

\section{Introduction}

Growth in the field of biosensors has been phenomenal. ${ }^{[1]}$ Single molecule techniques, which allow analyte molecules to be examined individually, ${ }^{[2]}$ have been showing great potential to meet the stringent requirements of both fundamental research and future point of care devices. Among these novel sensing techniques, nanopore based biosensors have emerged as an exciting possibility 
and have been applied to a variety of biological systems such as viral genomes, ${ }^{[3]}$ disease related DNA or RNA, ${ }^{[4-6]}$ antibodies,${ }^{[7]}$ transcription factors ${ }^{[8]}$ and other proteins. ${ }^{[9]}$ Instead of modifying the nanopore surface, another approach using DNA molecules as information carriers has shown potential as a highly adaptable sensing platform. Both small carriers of tens of base pairs ${ }^{[5,10]}$ and long molecules of few thousand base pairs ${ }^{[11-12]}$ have been shown with potential towards multiplexed sensing. The key idea is to program a long double-stranded DNA (carrier) with specific binding sites and a barcode for identification. ${ }^{[13]}$ Specific features in the ionic current signal confirm the presence of the target when the DNA carrier with the corresponding binding site passes through the nanopore. In previous studies, basic sensing functions including target identification, concentration quantification in the nanomolar range ${ }^{[14]}$ and real time kinetics screening ${ }^{[15]}$ have been demonstrated, and multiplexed detection of high affinity antibodies was

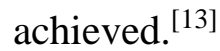

Until now, the nanopore based DNA carrier approach has relied on measurements in high salt concentrations of up to $4 \mathrm{M} \mathrm{LiCl}$. The high salt conditions enhance the signal to noise ratio ${ }^{[16]}$ and slow down the target translocation. ${ }^{[17]}$ However, the specific binding of many relevant biological targets is destabilized in these conditions. Although measurements in lower concentration buffers can be achieved, ${ }^{[12]}$ the sensing resolution and reading of detailed information encoded in the DNA carrier is severely limited. In this work, we demonstrate a two-step assay combining aptamer-based DNA circuits with our nanopore DNA carrier method to detect targets beyond tight binding proteins and antibodies. In Step 1, an aptamer-based DNA circuit is used to translate information about the target into an output DNA strand. In Step 2, the output DNA strand is read by nanopore sensing via a sequence specific DNA strand displacement reaction on the DNA carrier. Separating the process into two steps allows the bio-interactions to be carried out in adjustable physiological 
conditions and the signal translation enables the detection of small targets which cannot produce a distinguishable secondary current drop by attaching to the DNA carrier directly. In the subsequent sensing step, we are able to use buffers with high salt concentration to maximize the readout resolution.

\section{Results and Discussions}

\subsection{Design of the two-step sensing scheme}

As a first demonstration of our two-step sensing scheme we chose adenosine triphosphate (ATP) as the target. Aptamer tuned DNA strand displacement ${ }^{[18]}$ was adapted to translate the ATP signal into an output strand. ${ }^{[19-21]}$ In Figure 1a (Step 1), the ATP binding aptamer and toehold binding domain (ABA) are shown in blue and orange, respectively. After incubation at $37^{\circ} \mathrm{C}$ for two hours, the ABA strand forms a G-quadruplex upon binding to ATP molecules in the sample. This change in conformation inhibits the hybridization of $\mathrm{ABA}$ to the substrate, resulting in an open toehold $\mathrm{A}$ (orange) on the substrate. The input strand containing the complementary sequence of toehold A will then initiate the strand displacement reaction and thus release the output strand. In the case of no ATP in the sample, the input strand will not be able to release the output strand due to the closed toehold A.

(a) Step 1: signal translation from target tp output strand

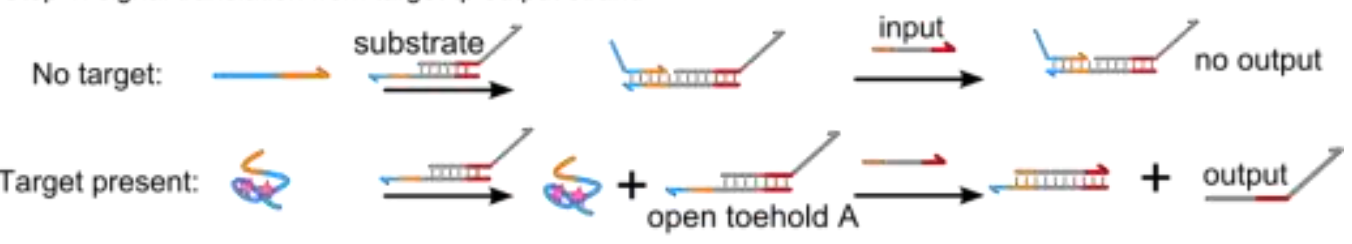

(b) Step 2: read output with nanopore
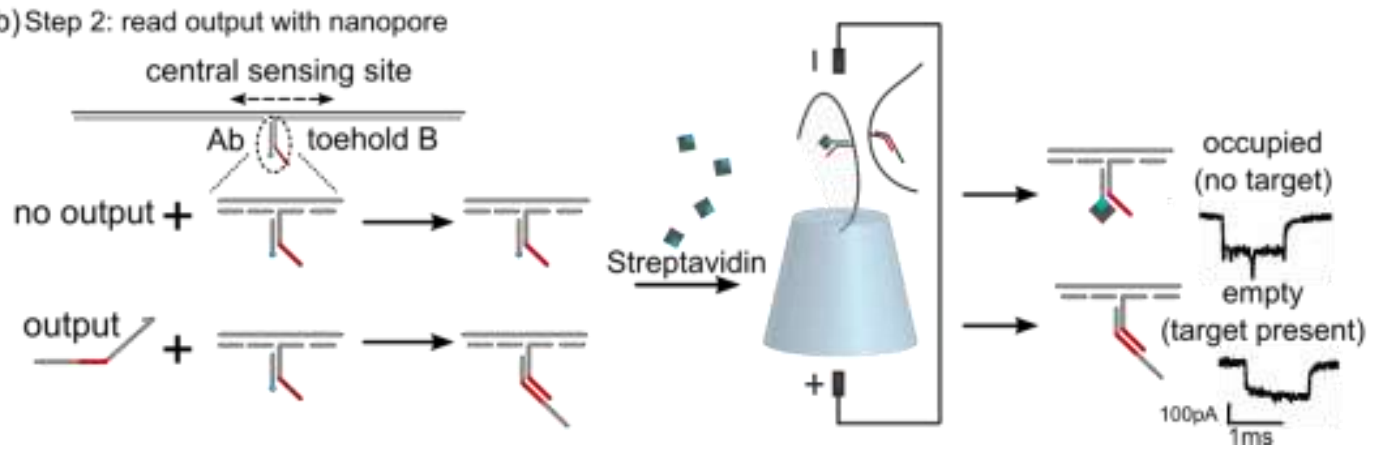
Figure 1. Schematic presentation of the two-step assay design. (a) Aptamer-based signal translation using a specifically designed DNA circuit (Step 1 of the sensing scheme). (b) Nanopore-based readout of the output strand. Example events are shown after incubation with monovalent streptavidin (blue-grey rectangles) for 'occupied' or 'empty' DNA carriers where the biotinylated strand is removed by the output strand. The blue rectangle represents the only active subunit of the monovalent streptavidin molecule.

In the second step (Figure 1b), DNA carriers functionalized with a specific toehold B (red) were used to examine the presence of the output strand and hence for ATP detection. Following our previous protocols, ${ }^{[11]}$ the DNA carriers were constructed using a 7228 bases long linear singlestranded 'scaffold' hybridized to 190 short (38 bases) synthetic DNA oligonucleotides. Only one sensing site was placed at the centre of the DNA carrier for establishing the sensing protocol. At the sensing site, a specific toehold probe is designed presenting an accessible overhang. A biotin labelled (blue spot) probe $\mathrm{Ab}$ is initially hybridized to part of the toehold probe and the toehold B is left free. Containing the complementary sequence to toehold $\mathrm{B}$, the output strand can efficiently initiate the displacement reaction and eventually remove the biotin labelled $\mathrm{Ab}$ from the sensing site. Therefore, the monovalent streptavidin ${ }^{[22]}$ added cannot bind and produce the secondary current drop signal in the ionic current signal. As shown in the example translocation events (Figure 1b), the 'empty' event without a secondary current drop corresponds to a DNA carrier without a biotin label and hence signals the presence of ATP. In the case of absence of ATP output strand, the translocation event appears 'occupied'. The fraction of empty events is the indicator for the existence of the output strand in the sample. It is important to notify that with our DNA carrier approach no modification of nanopores is necessary as the information is stored in the analytes.

\subsection{Characterization of the two-step sensing scheme}


We first examined how efficiently empty and occupied events can be differentiated. DNA carriers with biotin labelled probe $\mathrm{Ab}$ or the output strand added directly in the carrier synthesis were measured with the same nanopore for comparison. Figure 2a shows the peak current drop amplitude distributions of the two carriers. Fitted by a Gaussian function, the main peaks from the empty and occupied samples are $-0.15 \pm 0.01 \mathrm{nA}$ and $-0.18 \pm 0.01 \mathrm{nA}$ (mean \pm s.d., the same format in other parts), demonstrating two well resolved populations. As the smaller peak represents the current drop level of bare dsDNA, the ratio of $\sim 1.2$ between the two peaks is used as a threshold factor to define an occupied event in our analysis. A smaller peak is also shown in the distribution of the occupied carriers (Figure 2a, right), which is most likely caused by imperfect DNA carriers missing the biotin label. A constant background of around $10 \%$ is present in our previous work. ${ }^{[14]}$

(a)

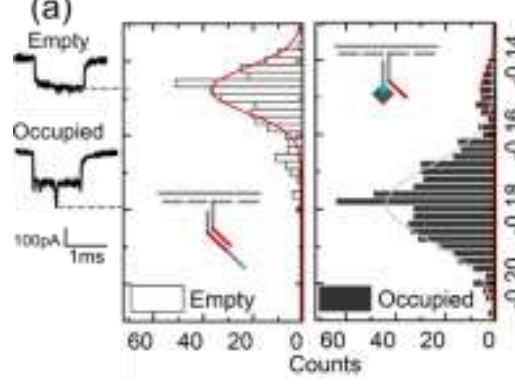

(b)

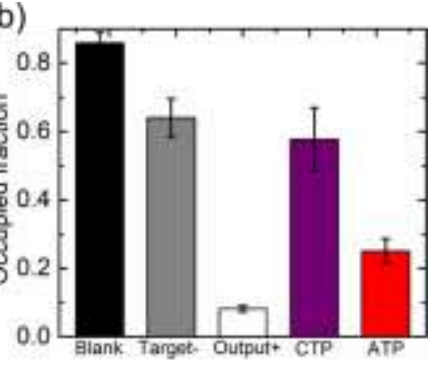

Figure 2. Proof-of-concept of the two-step assay with the ATP-aptamer system. (a) Peak current drop distributions of occupied and empty DNA carrier translocation events. The histograms were fitted by a Gaussian function. The event numbers for occupied and empty samples are 669 and 365, respectively. (b) ATP detection using the two-step assay. The Blank control (black) was measured with labelled DNA carriers before any reactions. The 'Target-' (grey) and 'Output+' (white) controls were measured by incubating the DNA carriers with ATP free DNA circuits or excess amount of output strands. The CTP experimental group (purple) was to demonstrate the specificity of the DNA circuit. The data contains 4143 events measured with 17 nanopores. 
Two control conditions were introduced to benchmark the readout fraction range of the occupied fraction readout (Figure 2b): output strands with 20 times molar concentration of the DNA carrier were incubated with the carrier to achieve the 'output + ' result. For the 'target-' control we incubated the carriers with ATP free DNA circuit. Occupied fractions of these two controls read at $0.08 \pm 0.01$ and $0.64 \pm 0.06$ respectively. The lower limit is likely caused by the presence of DNA folds or knots ${ }^{[23]}$ in the carrier that produce similar signals as the streptavidin label. The upper limit levels off at 0.64 , lower than the expected occupied fraction of 1 , which we attribute to two reasons. First, a small fraction of imperfect carriers without biotin labels is unable to contribute to the occupied fraction, as shown in Figure 2a. Second, a small amount of free or 'leaked' output strands may initiate strand displacement even in the absence of ATP. The occupied fraction of blank DNA carriers sample without any incubation is measured as another control. The occupied fraction of $0.86 \pm 0.03$ indicates that the initial reduction from 1 to $\sim 0.86$ is probably due to the imperfect DNA carriers while the rest from $\sim 0.86$ to $\sim 0.64$ is probably from the 'leaked' output. Better purification of the DNA circuits components and optimized designs may improve the readout fraction range. ${ }^{[24-25]}$

The occupied fraction with the ATP positive sample (Figure $2 b$, red) is $0.25 \pm 0.04$, showing a drop by $61 \%$ in occupied fraction compared to the 'target-' control. Reference electrophoresis assay is shown in Figure S4. CTP, GTP, TTP and UTP with the same concentration were measured to examine the ATP specificity of the designed DNA circuit (Figure S5). For CTP, the occupied fraction (Figure $2 \mathrm{~b}$, purple) we measured is $0.57 \pm 0.09$, only $11 \%$ lower than the 'target-' control. Our results demonstrate a successful detection of ATP with high specificity. It has to be noted that the 'ATP' occupied fraction (0.25) is higher than the lower limit (0.08), as the displacement reaction was probably not fully complete. Although higher concentration ratios between DNA 
circuits and carriers could help to reach a more complete reaction, the background signal increases in parallel. ${ }^{[15]}$ We use the concentration ratio of 10 in all the measurements to balance between high response with low background.

\subsection{Protein based barcoding}

In order to read multiple output strands simultaneously, we designed DNA carriers with a 'coding' region in the first half and a 'sensing' site in the middle of the second half (Figure 3b). The 'coding region' was constructed by having a protein attached as an individual sensing bit. Streptavidin and digoxigenin antibodies (anti-dig) were used to realize the digital readout given their tolerance to high salt conditions. ${ }^{[14]}$ Precisely located binding of the protein bits was achieved by modifying the DNA oligo in the desired location with a biotin or two digoxigenin labels. Due to the different size of the two protein molecules, secondary current drops of two levels were observed in the translocation events (Figure $3 \mathrm{~b}$ ): small secondary peaks from streptavidin $(\sim 53$ $\mathrm{kDa})$ are read as ' 0 ' and the anti-dig $(150 \mathrm{kDa})$, corresponding to the larger peaks, indicate ' 1 '. The distribution of current drop levels of the ' 0 ' and ' 1 ' bits shows two well separated populations (Figure S1). Figure $3 \mathrm{~b}$ shows the library of the 3 bits encoded DNA carriers. To verify the barcode reading accuracy, the library of 8 encoded carriers were mixed with the same molar concentration and measured in the same nanopore. Roughly equal quantities are observed for each barcode within pipetting errors (Figure S2). The use of single proteins yields adequate digital signals while simplifies the design of the coding region compared to an earlier strategy. ${ }^{[13]}$ 

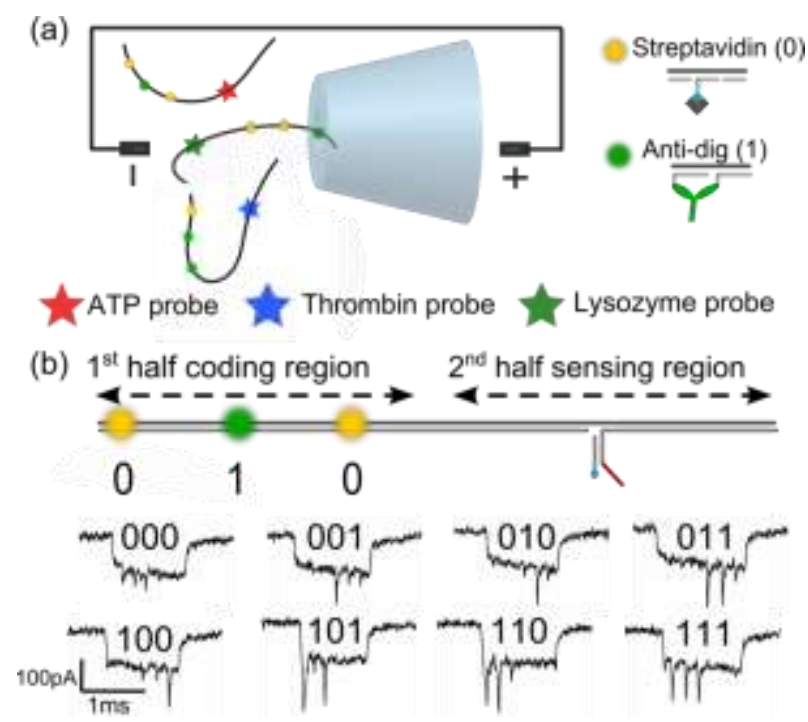

Figure 3. Digitally encoded DNA carrier designs using proteins. (a) Schematic presentation of the multiplexed DNA carrier-nanopore sensing platform. Selectivity is introduced by assigning the carrier with a specific barcode for each target system. (b) Designed DNA carriers consist of a coding region and a sensing region. Attached streptavidin (bit=0, yellow spot) and anti-dig (bit=1, green spot) produce secondary current drops of two amplitude levels. The coding region can be found either in the first or second half of the event depending on the orientation of the DNA carrier when entering the nanopore.

\subsection{Multiplexed sensing capability of the two-step sensing scheme}

Two other targets, thrombin and lysozyme, were added to demonstrate the multiplexed capability of the two-step assay (Figure 3a). With our protein coding, we assigned the barcodes 010, 011 and 100 to ATP, thrombin and lysozyme system respectively (Figure 4b). The barcode 001 is designed with an anti-dig bound to the sensing site as a referential marker. For the other 3 DNA carriers, the sensing site was designed in the same fashion as the strand displacement sensing site used for ATP. Each encoded DNA carrier has a toehold region in the overhang probe that reacts only to the output strand from its specific target. The DNA circuits for the three targets were designed to follow the same pattern and the experimental protocols were exactly the same as for 
ATP detection. All three DNA circuits were mixed together in the experimental groups. Results of our multiplexed measurements are shown in Figure 4c.

The drop in occupied fraction (drop\%) is used to indicate signal changes due to the target presence. Since the '001' carrier was fully labelled and measured in the same nanopore, its occupied fraction was set as 1 , yielding an internal reference in these measurements. The percentage drop of the occupied fraction from the reference in each measurement can then be used to determine whether a specific target exists in the sample or not. As shown in Figure 4c, 'output+' and 'target-' controls were carried out by incubating the mixed DNA carriers with three output strands or target free DNA circuits. The three targets were added individually or together in four experimental groups. The drop $\%$ threshold of $30 \%$ is used to determine a positive detection. We marked values above the threshold in bold. Expected positive detections are shaded in corresponding colours for the three systems. Our results show a perfect match between the expected and detected targets. Our multiplexed assays successfully detected all 3 targets individually and simultaneously. Each control and experimental group was measured with several nanopores (p1-p17). 
(a)

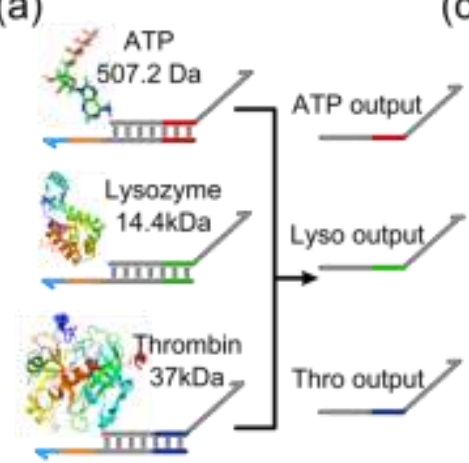

(b)

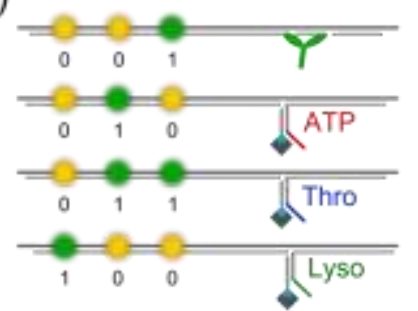

(d)

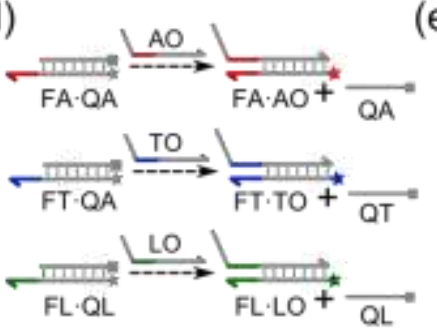

(c)

\begin{tabular}{|c|c|c|c|c|}
\hline $\begin{array}{l}\text { Sample } \\
\text { group }\end{array}$ & $\begin{array}{l}\text { Pore } \\
\text { No. }\end{array}$ & $\begin{array}{l}010 \\
\text { (drop\%) }\end{array}$ & $\begin{array}{l}011 \\
\text { (drop\%) }\end{array}$ & $\begin{array}{l}100 \\
\text { (drop\%) }\end{array}$ \\
\hline \multirow{2}{*}{$\begin{array}{l}\text { Output+ } \\
\text { control }\end{array}$} & $\mathrm{p} 1$ & 33.3 & 47.8 & 53.3 \\
\hline & p2 & 47.0 & 58 & 61 \\
\hline \multirow{2}{*}{$\begin{array}{l}\text { Target- } \\
\text { control }\end{array}$} & p3 & 0 & 23.1 & 17.6 \\
\hline & $p 4$ & 10.9 & 17.4 & 23.9 \\
\hline \multirow[t]{3}{*}{ ATP4 } & ps & 40.0 & 21.1 & 17.8 \\
\hline & p6 & 37.3 & 18.1 & 3.6 \\
\hline & p7 & 41.9 & 24.4 & 18.6 \\
\hline \multirow[t]{3}{*}{ Thro + } & p8 & 24.0 & 58.3 & 14.6 \\
\hline & p9 & 0 & 31.5 & 2.7 \\
\hline & p10 & 4.3 & 41.3 & 8.7 \\
\hline \multirow[t]{3}{*}{ Lysot } & p11 & 8.4 & 28.9 & $54: 2$ \\
\hline & 012 & 9.4 & 28.2 & 51.1 \\
\hline & p13 & 16.1 & 19.4 & 64.5 \\
\hline \multirow{4}{*}{$\begin{array}{l}\text { All } \\
\text { three+ }\end{array}$} & p14 & 33,7 & 70.9 & 53.5 \\
\hline & p1s & 61.5 & 68.1 & 54.2 \\
\hline & P16 & 46.5 & 48. 3 & 54.7 \\
\hline & p17 & 51.1 & 55.4 & 54.4 \\
\hline
\end{tabular}

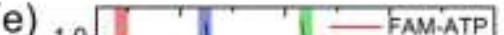

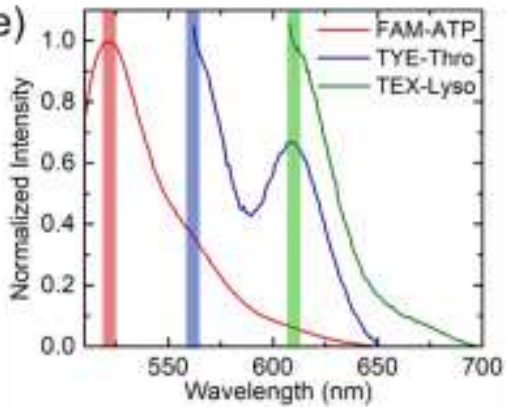

Figure 4. Multiplexed two step sensing using 3 aptamer-based systems. (a) Three targets with molecular weights between a few $100 \mathrm{Da}$ (ATP) and a few $10 \mathrm{kDa}$ (thrombin and lysozyme). (b) The complete designs of three specific DNA carrier structures with different barcodes. '010', '011' and '100' are assigned to ATP, thrombin and lysozyme respectively. (c) DNA carrier-nanopore results of the multiplexed samples. The percentage drop of the occupied fraction from the reference in each measurement is used here to indicate the signal changes due to the target presence. The two controls were measured by incubating DNA carriers with the 3 output strands (Output+) or target free DNA circuits (Target-). Drop\% above 30\% is marked in bold and the expected positive detection is shaded in red, blue and green for ATP, Thrombin and Lysozyme accordingly. 3365 assigned events from 17 different nanopores were considered in this table. (d) Fluorescent- 
quencher probe pairs used in the reference bulk assay. The 3 colours correspond to the three target systems. (e) Fluorescent spectrum when all 3 targets present in the sample.

In order to compare the performance of the nanopore-DNA carrier readout and traditional bulk measurements, we carried out a standard fluorescent-based assay. A pair of fluorescent and quencher probes was adapted from the overhang probes on the carrier sensing sites. As shown in Figure $4 \mathrm{~d}$, the fluorophore is initially quenched in the DNA duplex. Upon the presence of the output strand $\mathrm{AO}$, TO or LO, the same displacement as on the DNA carrier sensing site removes the quencher strand QA, QT or QL. However, the readout signal is the recovered fluorescent intensity instead. Multiplexed sensing was achieved by using 3 different fluorophores for the 3 targets: FAM for ATP, TYE-563 for thrombin and TEX-615 for lysozyme. Excited separately at the corresponding wavelengths (495 nm for FAM, $549 \mathrm{~nm}$ for TYE-563 and $596 \mathrm{~nm}$ for TEX615), the fluorescent spectra of a sample containing all 3 targets are shown in Figure 4e. Fluorescent signals were observed at the respective maximum emission wavelengths of the three dyes (520 nm, $563 \mathrm{~nm}$ and $615 \mathrm{~nm}$ accordingly), which indicated the presence of all 3 targets in the sample. Additional fluorescent results of the other experimental groups are shown in Figure S6. To investigate the effect of high salt concentration on the target recognition in step 1, fluorescence measurements in buffers with and without $4 \mathrm{M} \mathrm{LiCl}$ were compared (Figure S7) and results show that high salt concentration disrupts the binding for all 3 targets to their aptamer sequences. Although fluorophores with well separated excitation and emission wavelengths were used, signals show significant overlap in the fluorescence-based assay. For example in Figure 4e, the signal of TEX-615 is observed within the emission of TYE-563, which is due to the higher fluorescence quantum yield of TEX-615 compared to TYE-563. Spectral overlap and varied 
fluorescence quantum yields can cause difficulties in understanding the multiplexed fluorescence data and set strict limits to the number of simultaneous targets. ${ }^{[26-28]}$

\section{Conclusion}

In summary, we have presented a two-step assay towards multiplexed and single molecule sensing in adjustable physiological conditions. In Step 1, the target signal is translated into an output DNA strand using an aptamer-based DNA circuit, while in Step 2, the output strand is read by a strand displacement designed on the DNA carrier. The separate Step 1 provides the biological reaction with optimal physiological conditions. The translation of the signal into an output DNA strand allows for the detection of molecules ranging from a few hundred Daltons (ATP) up to tens of kilo Daltons using nanopore sensing in high ionic strength buffer. We also demonstrate the multiplexing capability of the developed sensing scheme, 3 bits protein encoded DNA carriers were used to simultaneously detect 3 target molecules that vary in size and affinity. The total assay time required is $\sim 1.5$ hours for step 1 and $\sim 4$ hours in step 2 , with an active handling time of around 1 hour. Multichannel nanopore recordings can be applied to reduce the time required in step 2. Our two-step assay has built a bridge between the nanopore-based single-molecule method and DNAbased circuitry ${ }^{[29-30]}$ designs. Apart from the aptamer-based conformation changes used in this work, direct binding of antibodies and other proteins can also facilitate successful signal translation into output strands. ${ }^{[1-33]}$ Compared to the traditional fluorescence assays, our method avoids fluorophores conjugation and spectra overlap. Furthermore, sensing performance can be further enhanced for example by signal amplification via enzyme free hybridization chain reactions, ${ }^{[34-35]}$ in Step 1. Throughput can be further improved in both the DNA carrier design and nanopore resolution. Under current experimental conditions, the minimum spacing between two resolved secondary current drops is around $200 \mathrm{bp}$ (Figure S3). Given the $7.2 \mathrm{kbp}$ length of the DNA carrier,

more than 10 bits can be fit in the coding region, providing a carrier library of $2^{10}=1024$. In case 
more targets are necessary, thin membrane nanopore devices made from $\mathrm{SiN}^{[36-37]}$ and 2dimensional materials ${ }^{[38-40]}$ should increase the number of readable bits on the carrier.

\section{Experimental Section}

\section{DNA carrier synthesis}

DNA carriers were constructed using a 7228 bases long linear single-stranded 'scaffold' hybridized to 190 short synthetic DNA oligonucleotides. The length of each DNA oligo is 38 bases. The hybridization was carried out by heating up the mixture of the scaffold (20 nM) and oligos (60 $\mathrm{nM}$ each) to $70^{\circ} \mathrm{C}$ and then cooled down linearly to $25^{\circ} \mathrm{C}$ over 1 hour. A concentration of 120 $\mathrm{nM}$ was used for all modified oligos in the mixture. $14 \mathrm{mM} \mathrm{MgCl} 2$ buffered with TE was used in this hybridization. The DNA carriers were then purified using Amicon Ultra $100 \mathrm{kDa}$ cutoff centrifugal filters. Three centrifuge rounds of $3000 \mathrm{~g}$ at $4^{\circ} \mathrm{C}$ were carried out, each lasting for 10 minutes. The modified oligo sequences for both sensing and coding region are shown in Table S1 and Table S2. Full sequences of the 190 short DNA oligos can be found in our previous papers. $^{[11,13]}$

\section{Nanopore fabrication and experimental setup}

Glass nanopores used in this work were fabricated with a laser-assisted puller (P-2000, Sutter Instrument, USA). Details of the nanopore geometry including SEM images and a 3D model with two taper angles are as described previously. ${ }^{[11,13]}$ The current drop amplitude contributed by the bare double-stranded DNA (dsDNA level) was used to indicate the nanopore size. The dsDNA level is averaged at $-0.129 \pm 0.019 \mathrm{nA}$ over the 43 nanopores used in this paper. Ionic current measurements were carried out using a standard patch clamp amplifier (Axopatch 200B, Molecular Devices, USA). $600 \mathrm{mV}$ bias voltage was applied in all the measurements at the sampling frequency of $250 \mathrm{kHz}$. The data was filtered at $49.9 \mathrm{kHz}$ with an external 8-pole Bessel filter. 


\section{Experimental protocol of the two-step assay}

Step1: The M13mp18 DNA scaffold was purchased from New England BioLabs and all DNA oligos were ordered from Integrated DNA Technologies. $100 \mathrm{mM} \mathrm{NaCl}, 10 \mathrm{mM} \mathrm{KCl}$ and $10 \mathrm{mM}$ $\mathrm{MgCl}_{2}$ buffered with $10 \mathrm{mM}$ Tris- $\mathrm{HCl}, \mathrm{pH} 8$ was used in all incubations, where $\mathrm{K}^{+}$was included to help the formation of G-quadruplex structures. The substrate was hybridized by heating up the two strands to $88^{\circ} \mathrm{C}$ for 5 minutes and then cooling down linearly to room temperature over 30 minutes. The ABA hybridization and input strand displacement were carried out at room temperature for 1 hour. The concentrations of the DNA circuit (ABA-substrate) and ATP (Thermo Fisher Scientific) used in step 1 are $100 \mathrm{nM}$ and $300 \mu \mathrm{M}$, while $120 \mathrm{nM}$ input strands were added to ensure maximum release of the output strands. For other target systems, $60 \mathrm{nM}$ Thrombin (Haematologic Technologies) and $29 \mu \mathrm{M}$ lysozyme (Sigma-Aldrich) were used in the incubations. Quantitative concentration measurements were not carried out in this work, however the feasibility of measuring concentrations has been demonstrated in our previous work. ${ }^{[14,15]}$ Gel electrophoresis was used as reference assay to validate the structural changes in the DNA circuit (Figure S4).

Step 2: DNA circuits of $3 \mathrm{nM}$ were incubated with $0.3 \mathrm{nM}$ DNA carriers for 2 hours at room temperature to reach the 'complete' state of the displacement reaction. ${ }^{[14]}$ Given the sample volume of $\sim 10 \mu \mathrm{L}$ required in our device, ${ }^{[41]}$ the total amount of DNA carrier is only 3 femtomolar. As for the multiplexed measurements, equal amounts of 4 encoded DNA carries were mixed to make a total concentration of $0.3 \mathrm{nM}$. The incubation buffer was the same as in Step 1, while $4 \mathrm{M} \mathrm{LiCl}$ was only added for the nanopore measurements. $4.8 \mathrm{nM}$ anti-dig were added $10 \mathrm{~min}$ before the measurements together with $2.4 \mathrm{nM}$ streptavidin to amplify the secondary signals in both coding region and sensing region.

\section{Supporting Information}

Supporting Information is available from the Wiley Online Library or from the author. 


\section{Acknowledgements}

The authors thank the Howarth Lab Oxford for the monovalent streptavidin. J. Cama and J. Mc Hugh for critical reading the manuscript. J. Kong acknowledges funding from Cambridge Trust. J. Zhu acknowledges funding from EPSRC grant (EP/M008258/1). K. Chen and U. F. Keyser acknowledge funding from an ERC Consolidator grant (DesignerPores 647144).

Received: ((will be filled in by the editorial staff))

Revised: ((will be filled in by the editorial staff)) Published online: ((will be filled in by the editorial staff))

References

[1] A. P. Turner, Chem Soc Rev. 2013, 42, 3184.

[2] J. J. Gooding, K. Gaus, Angew Chem Int Edit. 2016.

[3] A. Singer, S. Rapireddy, D. H. Ly, A. Meller, Nano Lett. 2012, 12, 1722.

[4] M. Wanunu, T. Dadosh, V. Ray, J. Jin, L. McReynolds, M. Drndić, Nat Nanotechnol. $2010,5,807$.

[5] O. K. Zahid, B. S. Zhao, C. He, A. R. Hall, Sci Rep. 2016, 6, 29565.

[6] Z. Zhu, Y. Zhou, X. Xu, R. Wu, Y. Jin, B. Li, Anal Chem. 2017, 90, 814.

[7] E. C. Yusko, J. M. Johnson, S. Majd, P. Prangkio, R. C. Rollings, J. Li, J. Yang, M.

Mayer, Nat Nanotechnol. 2011, 6, 253.

[8] A. Squires, E. Atas, A. Meller, Sci Rep. 2015, 5, 11643.

[9] R. Wei, V. Gatterdam, R. Wieneke, R. Tampé, U. Rant, Nat Nanotechnol. 2012, 7, 257.

[10] E. Beamish, V. Tabard-Cossa, M. Godin, ACS sensors. 2017.

[11] N. A. Bell, U. F. Keyser, J Am Chem Soc. 2015, 137, 2035.

[12] J. Y. Sze, A. P. Ivanov, A. E. Cass, J. B. Edel, Nat Commun. 2017, 8, 1552.

[13] N. A. Bell, U. F. Keyser, Nat Nanotechnol. 2016, 11, 645.

[14] J. Kong, N. A. Bell, U. F. Keyser, Nano Lett. 2016, 16, 3557.

[15] J. Kong, J. Zhu, U. F. Keyser, Chem Commun. 2017, 53, 436. 
[16] S. Ghosal, Phys Rev Lett. 2007, 98, 238104.

[17] S. W. Kowalczyk, D. B. Wells, A. Aksimentiev, C. Dekker, Nano Lett. 2012, 12, 1038.

[18] Y. Du, S. Dong, Anal Chem. 2016, 89, 189.

[19] J. H. Monserud, K. M. Macri, D. K. Schwartz, Angew Chem Int Edit. 2016, 55, 13710.

[20] J. Zhu, L. Zhang, Z. Zhou, S. Dong, E. Wang, Chem Commun. 2014, 50, 3321.

[21] Y. Xing, Z. Yang, D. Liu, Angew Chem Int Edit. 2011, 50, 11934.

[22] M. Howarth, D. J. Chinnapen, K. Gerrow, P. C. Dorrestein, M. R. Grandy, N. L.

Kelleher, A. El-Husseini, A. Y. Ting, Nat Methods. 2006, 3, 267.

[23] C. Plesa, D. Verschueren, S. Pud, J. van der Torre, J. W. Ruitenberg, M. J. Witteveen, M. P. Jonsson, A. Y. Grosberg, Y. Rabin, C. Dekker, Nat Nanotechnol. 2016, 11, 1093.

[24] A. J. Thubagere, C. Thachuk, J. Berleant, R. F. Johnson, D. A. Ardelean, K. M. Cherry, L. Qian, Nat Commun. 2017, 8, 14373.

[25] D. Y. Zhang, E. Winfree, Nucleic Acids Res. 2010, 38, 4182.

[26] J. R. Epstein, I. Biran, D. R. Walt, Anal Chim Acta. 2002, 469, 3.

[27] K. D. Bake, D. R. Walt, Annu. Rev. Anal. Chem. 2008, 1, 515.

[28] W. F. An, in Cell-Based Assays for High-Throughput Screening, Springer, 2009, 97.

[29] J. Liu, Z. Cao, Y. Lu, Chem Rev. 2009, 109, 1948.

[30] D. Li, S. Song, C. Fan, Acc Chem Res. 2010, 43, 631.

[31] S. Ranallo, M. Rossetti, K. W. Plaxco, A. Vallée - Bélisle, F. Ricci, Angew Chem. 2015, $127,13412$.

[32] S. Ranallo, C. Prévost-Tremblay, A. Idili, A. Vallée-Bélisle, F. Ricci, Nat Commun. $2017,8,15150$. 
[33] W. Engelen, L. H. Meijer, B. Somers, T. F. De Greef, M. Merkx, Nat Commun. 2017, 8, 14473.

[34] R. M. Dirks, N. A. Pierce, Natl Acad Sci U. S. A. 2004, 101, 15275.

[35] B. Li, A. D. Ellington, X. Chen, Nucleic Acids Res. 2011, 39, e110.

[36] A. Singer, M. Wanunu, W. Morrison, H. Kuhn, M. Frank-Kamenetskii, A. Meller, Nano Lett. 2010, 10, 738 .

[37] K. Briggs, H. Kwok, V. Tabard - Cossa, Small. 2014, 10, 2077.

[38] G. F. Schneider, S. W. Kowalczyk, V. E. Calado, G. Pandraud, H. W. Zandbergen, L. M. Vandersypen, C. Dekker, Nano Lett. 2010, 10, 3163.

[39] C. A. Merchant, K. Healy, M. Wanunu, V. Ray, N. Peterman, J. Bartel, M. D. Fischbein, K. Venta, Z. Luo, A. C. Johnson, Nano Lett. 2010, 10, 2915.

[40] K. Liu, J. Feng, A. Kis, A. Radenovic, ACS Nano. 2014, 8, 2504.

[41] N. A. Bell, V. V. Thacker, S. Hernández-Ainsa, M. E. Fuentes-Perez, F. Moreno-Herrero, T. Liedl, U. F. Keyser, Lab Chip. 2013, 13, 1859. 
The multiplexed biosensing with high specificity and accuracy is of fundamental importance in biological research and point of care diagnostics. Combining aptamer based DNA circuits and protein encoded DNA carriers, we present a two-step assay that expands the working range beyond just tight binding model systems. The results demonstrate a simultaneous detection of 3 targets varies in molecular weight and affinity.

Keyword: Nanopore, Aptamer, Multiplexed sensing, DNA carrier, Physiological condition

J. Kong, J. Zhu, K. Chen and U. F. Keyser*

Specific biosensing using DNA aptamers and nanopores

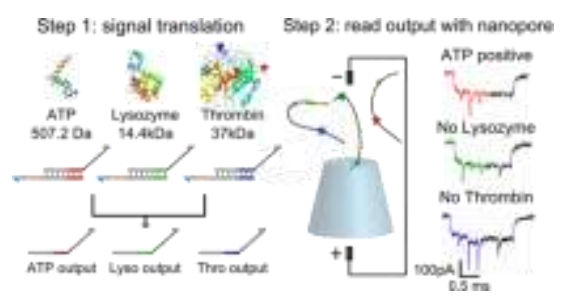

